\title{
Damage Tensors and Tensors of Continuity in Stress Analysis
}

\author{
J. Betten \\ Technical University Aachen \\ Z. Naturforsch. 38 a, $1383-1390$ (1983); received October 17, 1983*
}

Starting from a third order skew-symmetric tensor of continuity to represent area vectors (bivectors) of Cauchy's tetrahedron in a damaged state, a second order damage tensor is found which has the diagonal form with respect to the considered coordinate system.

The second part of the paper is concerned with the stresses in a damaged continuum. Introducing a linear operator of rank four a net-stress tensor is formulated. This tensor can be decomposed into a symmetric part and into an antisymmetric one, where only the symmetric part is equal to the net-stress tensor introduced by Rabotnov [7].

In view of the formulation of constitutive equations the non-symmetric property of the actual net-stress tensor is a disadvantage. Therefore, a pseudo-net-stress tensor is introduced, which is symmetric.

\section{Introduction}

The creep process in its tertiary phase is essentially influenced by the effect of damage. It is well known that the process of creep of a metal is accompanied by the formation of microscopic cracks on the grain boundaries and that damage-accumulation occurs.

In the phenomenological uni-axial theory due to Kachanov (1958) material deterioration is considered by introducing an additional variable $\omega$ or, alternatively, $\psi \equiv 1-\omega$ into the constitutive equations i.e. the strain rate can be expressed in the form $\dot{\varepsilon}=f(\sigma, \psi)$, where $\sigma$ is the nominal stress of a uniaxial tension specimen. The material parameters $\omega$ and $\psi$ describe the current state of material damage and the "continuity" of the material, respectively. The parameter of "continuity", $\psi$, represents that fraction of the cross-sectional area which is not occupied by either voids or internal fissures. The net stress acting over the cross-section of a uniaxial tension specimen is then $\hat{\sigma}=\sigma / \omega$. When $\psi=1$ the material is in its virgin state and the constitutive equation mentioned above is reduced to the corresponding relationship for the secondary creep stage. When $\psi=0$ the material can no longer sustain load and the function is required to approach an infinite

* The first version was received June 25, 1982.

Reprint requests to Prof. Dr.-Ing. J. Betten, Mathematische Modelle in der Werkstoffkunde, Techn. Hochschule Aachen, Templergraben 55, D-5100 Aachen. strain rate. Furthermore, it is assumed that the damage rate $\dot{\omega}$ or, alternatively, the rate of change of the continuity $\dot{\psi}$ is also governed by the nominal stress and the current state of continuity, i.e. $\dot{\psi}=-g(\sigma, \psi)$.

Deliberations about the forms of the functions $f$ and $g$ were made in detail by many scientists, for instance by Chrzanowski [1], Goel [2], Hayhurst and Leckie [3], Hayhurst et al. [4]. In generalisation of Kachanov's theory constitutive equations and anisotropic damage growth equations are given by expressions like

$$
\dot{\varepsilon}_{i j}=f_{i j}(\sigma, \omega) \text { and } \quad \dot{\omega}_{i j}=g_{i j}(\sigma, \omega),
$$

respectively, where $\boldsymbol{\sigma}$ is Cauchy's stress tensor, and $\omega$ is an anisotropic damage tensor. Creep damage tensors are constructed, for instance, by Murakami and Ohno $[5,6]$. They assumed that material damage accumulating in the process of creep can be expressed by a symmetric tensor of rank two. Rabotnov [7] introduced also a symmetric second order tensor of damage and defined a symmetric net stress tensor $\hat{\boldsymbol{\sigma}}$ by a linear transformation, $\sigma_{i j}=$ $\Omega_{i j k l} \hat{\sigma}_{k l}$, where the fourth order tensor $\boldsymbol{\Omega}$ is assumed to be symmetric. In the present paper it is pointed out that the fourth order tensor is only symmetric corresponding to the first index pair $i j$, but not to the second one $k l$. Thus, the net stress tensor is not symmetric in the anisotropic damage case.

Starting from a third order skew-symmetric tensor of continuity to represent area vectors (bivec- 
tors) of Cauchy's tetrahedron in a damaged state, we finally find a second order damage tensor which has the diagonal form.

\section{Definitions}

In this paper rectangular cartesian components of the tensors are used througout; results discussed here can be expressed in terms of general curvilinear coordinate systems by the standard techniques of tensor analysis. Index notation and Einstein's summation convention are employed. Confining to cartesian tensors we define:

In a $v$-dimensional space a (cartesian) tensor of valence $\mu$ alternating in all indices is called a $\mu$ vector, multivector, polyvector, antisymmatric tensor or skew-symmetric tensor,

$$
A_{k_{1} \ldots k_{\mu}} \equiv A_{\left[k_{1} \ldots k_{\mu}\right]}
$$

where $\mu \leqq v$.

Multivectors of orders $2,3,4, \ldots, \mu$ are also called bi-vectors, tri-vectors, quadri-vectors, $\ldots, \mu$-vectors, respectively. A skew-symmetric tensor possessesses exactly $\left(\begin{array}{l}v \\ \mu\end{array}\right) \equiv \frac{v !}{\mu !(v-\mu) !}$ non zero-independent and distinct (essentially distinct, i.e. differing not only in sign) components.

In the following we restrict to a three-dimensional space $(v=3)$ and consider bi-vectors and tri-vectors:

$A_{[i j]} \equiv\left(A_{i j}-A_{j i}\right) / 2$,

$A_{[i j k]} \equiv\left(A_{i j k}+A_{j k i}+A_{k i j}-A_{i k j}-A_{j i k}-A_{k j i}\right) / 6$.

If we consider skew-symmetric tensors with respect to $i<\mu$ indices, for instance

$$
T_{k_{1} \ldots k_{\mu}} \equiv T_{\left[k_{1} \ldots k_{\lambda}\right] k_{\lambda+1} \ldots k_{\mu}} \text { where } \lambda \leqq v,
$$

we have in $v$-dimensional space $\left(\begin{array}{l}v \\ \lambda\end{array}\right) \cdot v^{\mu-2}$ essential components. In (2.4) the operation of alternation is indicated by placing square brackets only around those indices to which it applies, that is, the $\lambda$ bracketed indizes $k_{1} \ldots k_{;}$are permutated in all possible ways, while indices which are excluded from the alternation are not bracketed. They keep their positions. Thus we obtain $\lambda !$ terms. The terms corresponding to even permutations are given a plus sign, those which correspond to odd permutations a minus sign, and they are then added and divided by $\lambda$ !. In three-dimensional space $\lambda$ can only be equal to two or to three.

\section{Geometry}

In three-dimensional space a parallelogram formed by the vectors $A_{i}$ and $B_{i}$ can be represented by

$$
S_{i}=\varepsilon_{i j k} A_{j} B_{k}
$$

or in the dual form

$$
S_{i j}=\varepsilon_{i j k} S_{k} \Leftrightarrow S_{i}=\frac{1}{2} \varepsilon_{i j k} S_{j k},
$$

where $\varepsilon_{i j k}$ is the third-order alternating tensor $\left(\varepsilon_{i j k}=1\right.$, or -1 accordingly as $i, j, k$ are even or odd permutations of $1,2,3$, respectively, otherwise the components $\varepsilon_{i j k}$ are equal to zero). From $(3.1 \mathrm{a}, \mathrm{b})$ we immediately find

$$
S_{i j}=2 ! A_{[i} B_{j]}=\left|\begin{array}{cc}
A_{i} & A_{j} \\
B_{i} & B_{j}
\end{array}\right| .
$$

Because of the decomposition (3.2) as an alternating product of two vectors the bi-vector $\boldsymbol{S}$ is called simple and has the following three nonvanishing essential components

$$
\begin{aligned}
& S_{12}=A_{1} B_{2}-A_{2} B_{1}, \quad S_{23}=A_{2} B_{3}-A_{3} B_{2}, \\
& S_{31}=A_{3} B_{1}-A_{1} B_{3} .
\end{aligned}
$$

In rectilinear components in three-dimensional space, we see that the absolute values of the components (3.3) are the projections of the area of the parallelogram, considered above, on the coordinate planes. Thus $S_{i j}$, according to (3.2), represents an area vector in three-dimensional space and has an orientation fixed by (3.1 a).

According to (3.1 b) a surface element $\mathrm{d} S$ with an unit normal $n_{i}$, i.e. $\mathrm{d} S_{i}=n_{i} \mathrm{~d} S$, is expressed by

$$
\mathrm{d} S_{i j}=\varepsilon_{i j k} \mathrm{~d} S_{k} \Leftrightarrow \mathrm{d} S_{i}=\frac{1}{2} \varepsilon_{i j k} \mathrm{~d} S_{j k}
$$

and

$$
n_{i j}=\varepsilon_{i j k} n_{k} \Leftrightarrow n_{i}=\frac{1}{2} \varepsilon_{i j k} n_{j k} .
$$

The components of the bi-vector $\boldsymbol{n}$ are the direction cosines $n_{1}, n_{2}, n_{3}$ :

$$
n_{i j}=\left(\begin{array}{ccc}
0 & n_{3} & -n_{2} \\
-n_{3} & 0 & n_{1} \\
n_{2} & -n_{1} & 0
\end{array}\right) .
$$


The principal invariants of (3.5), defined as

$$
\begin{aligned}
& J_{1} \equiv n_{i i}, \quad-J_{2} \equiv n_{i[i]} n_{j[j]}, \\
& J_{3} \equiv n_{i[i]} n_{j[j]} n_{k[k]},
\end{aligned}
$$

take the following values:

$$
\begin{array}{ll}
J_{1}(\boldsymbol{n})=0, & -J_{2}(\boldsymbol{n})=n_{1}^{2}+n_{2}^{2}+n_{3}^{2}=1, \\
J_{3}(\boldsymbol{n})=0, &
\end{array}
$$

i.e. the only nonvanishing invariant is determined by the length of the vector $n_{i}$.

Imagine that at a point $o$ in a continuous medium a set of rectangular coordinate axes is drawn and a differential tetrahedron is bounded by parts of the three coordinate planes through $o$ and a fourth plane not passing through $o$, as shown in Figure $1 \mathrm{a}$. Such a tetrahedron can be characterised by a system of bi-vectors,

$$
\begin{aligned}
& \mathrm{d}^{1} S_{i}=-\frac{1}{2} \varepsilon_{i j k}\left(\mathrm{~d} x_{2}\right)_{j}\left(\mathrm{~d} x_{3}\right)_{k}, \\
& \mathrm{~d}^{2} S_{i}=-\frac{1}{2} \varepsilon_{i j k}\left(\mathrm{~d} x_{3}\right)_{j}\left(\mathrm{~d} x_{1}\right)_{k}, \\
& \mathrm{~d}^{3} S_{i}=-\frac{1}{2} \varepsilon_{i j k}\left(\mathrm{~d} x_{1}\right)_{j}\left(\mathrm{~d} x_{2}\right)_{k}, \\
& \mathrm{~d}^{4} S_{i}=\frac{1}{2} \varepsilon_{i j k}\left[\left(\mathrm{~d} x_{1}\right)_{j}-\left(\mathrm{d} x_{3}\right)_{j}\right]\left[\left(\mathrm{d} x_{2}\right)_{k}-\left(\mathrm{d} x_{3}\right)_{k}\right],
\end{aligned}
$$

where the sum is the zero vector:

$$
\mathrm{d}^{1} S_{i}+\mathrm{d}^{2} S_{i}+\mathrm{d}^{3} S_{i}+\mathrm{d}^{4} S_{i}=O_{i} .
$$

In a damaged continuum we define a "net crosssection" $\hat{S} \equiv \psi S$ where $\psi \leqq 1$ describes the "continuity" of the material, as mentioned in the introduction. Then, by analogy of (3.8), a tetrahedron in a damaged continuum (Fig. 1 b) can be characterized by the following system of bi-vectors:

$$
\begin{aligned}
\mathrm{d}^{1} \hat{S}_{i} & =-\frac{1}{2} \alpha_{i j k}\left(\mathrm{~d} x_{2}\right)_{j}\left(\mathrm{~d} x_{3}\right)_{k} \equiv \alpha \mathrm{d}^{1} S_{i}, \\
\mathrm{~d}^{2} \hat{S}_{i}= & -\frac{1}{2} \beta_{i j k}\left(\mathrm{~d} x_{3}\right)_{j}\left(\mathrm{~d} x_{1}\right)_{k} \equiv \beta \mathrm{d}^{2} S_{i}, \\
\mathrm{~d}^{3} \hat{S}_{i}= & -\frac{1}{2} \gamma_{i j k}\left(\mathrm{~d} x_{1}\right)_{j}\left(\mathrm{~d} x_{2}\right)_{k} \equiv \gamma \mathrm{d}^{3} S_{i}, \\
\mathrm{~d}^{4} \hat{S}_{i}= & \frac{1}{2} \varkappa_{i j k}\left[\left(\mathrm{~d} x_{1}\right)_{j}-\left(\mathrm{d} x_{3}\right)_{j}\right] \\
& \cdot\left[\left(\mathrm{d} x_{2}\right)_{k}-\left(\mathrm{d} x_{3}\right)_{k}\right] \equiv \chi \mathrm{d}^{4} S_{i},
\end{aligned}
$$

where $\alpha_{i j k} \equiv \alpha \varepsilon_{i j k}, \beta_{i j k} \equiv \beta \varepsilon_{i j k}$, etc. are total skewsymmetric tensors of order three, which have the essential components $\alpha_{123} \equiv \alpha, \beta_{123} \equiv \beta$, etc., respectively.

From Fig. $1(\mathrm{a}, \mathrm{b})$ we find that only $\mathrm{d} S_{1}=-n_{1} \mathrm{~d} S$, $\mathrm{d}^{1} \hat{S}_{1}=\alpha \mathrm{d}^{1} S_{1}, \mathrm{~d}^{2} S_{2}=-n_{2} \mathrm{~d} S$, etc. are non vanishing components of the bi-vector systems (3.8) and
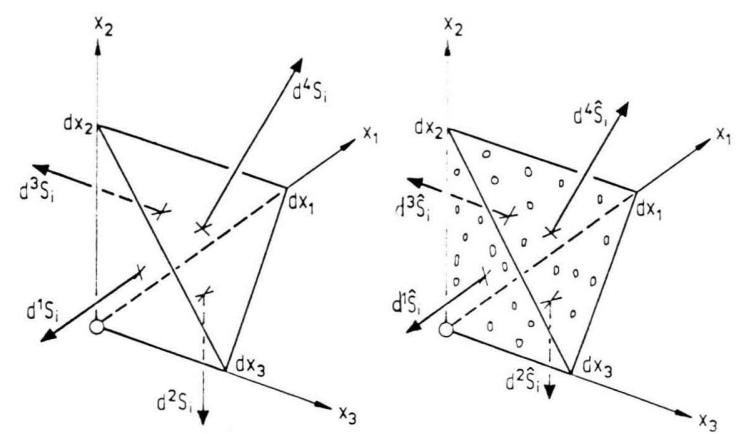

Fig. 1. Cauchy's tetrahedron a) in an undamaged state, b) in a damaged state.

(3.10). Then the sum of (3.10) yield the vector

$$
\Sigma_{i} \equiv \mathrm{d}^{1} \hat{S}_{i}+\ldots+\mathrm{d}^{4} \hat{S}_{i}=\left(\begin{array}{c}
(\varkappa-\alpha) n_{1} \\
(\varkappa-\beta) \\
n_{2} \\
(\varkappa-\gamma) n_{3}
\end{array}\right) \mathrm{d} S,
$$

which is not the zero-vector, unless in the isotropic damage case $(\alpha=\beta=\gamma=\chi)$ or in the undamaged case $(\alpha=\beta=\gamma=\chi=1)$ according to (3.9).

Furthermore, because of $\mathrm{d}^{1} \hat{S}_{1} \neq 0, \mathrm{~d}^{1} \hat{S}_{2}=\mathrm{d}^{1} \hat{S}_{3}=0$ etc., the damage state of the continuum at a point is characterized by the bivectors

$$
\begin{array}{cc}
\alpha_{1 i j}= & \left(\begin{array}{rrr}
0 & 0 & 0 \\
0 & 0 & \alpha \\
0 & -\alpha & 0
\end{array}\right), \quad \beta_{2 i j}=\left(\begin{array}{rrr}
0 & 0 & -\beta \\
0 & 0 & 0 \\
\beta & 0 & 0
\end{array}\right), \\
\gamma_{3 i j}=\left(\begin{array}{rrr}
0 & \gamma & 0 \\
-\gamma & 0 & 0 \\
0 & 0 & 0
\end{array}\right) .
\end{array}
$$

In the following we will examine if the bi-vector

$$
\begin{aligned}
& \psi_{i j}=\alpha_{1 i j}+\beta_{2 i j}+\gamma_{3 i j}=\left(\begin{array}{rrr}
0 & \gamma & -\beta \\
-\gamma & 0 & \alpha \\
\beta & -\alpha & 0
\end{array}\right) \\
& \psi_{i j}=\alpha \varepsilon_{1 i j}+\beta \varepsilon_{2 i j}+\gamma \varepsilon_{3 i j}
\end{aligned}
$$

could be a suitable "tensor of continuity". Then the damage tensor $\omega$ would be of the form

$$
\omega_{i j}=\delta_{(k) k} \varepsilon_{k i j}-\psi_{i j}=\left(\begin{array}{ccc}
0 & 1-\gamma & -(1-\beta) \\
-(1-\gamma) & 0 & 1-\alpha \\
1-\beta & -(1-\alpha) & 0
\end{array}\right)
$$


(no sum on the bracketed index $k$ ) or

$\omega_{i j}=(1-\alpha) \varepsilon_{1 i j}+(1-\beta) \varepsilon_{2 i j}+(1-\gamma) \varepsilon_{3 i j}$.

If a tensor is symmetric or antisymmetric, respectively, in one Cartesian coordinate system, it is symmetric or antisymmetric in all such systems; thus symmetry and antisymmetry are really tensor properties. Therefore, the skew-symmetric tensor ( $3.13 \mathrm{a})$ has only three essential components in any Cartesian system, for instance, $\alpha, \beta, \gamma$ in relation to the system $x_{i}$ or $\alpha^{*}, \beta^{*}, \gamma^{*}$ with respect of the system $x_{i}^{*}$.

The only nonvanishing invariants of the bi-vectors (3.13) and (3.14) are determined by their lengths:

$-J_{2}(\psi) \equiv-\frac{1}{2} \operatorname{tr} \psi^{2} \equiv-\frac{1}{2} \psi_{i j} \psi_{j i}=\alpha^{2}+\beta^{2}+\gamma^{2}$,

$-J_{2}(\omega)=(1-\alpha)^{2}+(1-\beta)^{2}+(1-\gamma)^{2}$.

In the undamaged state $(\alpha=\beta=\gamma=1)$ we have $-J_{2}(\psi)=3,-J_{2}(\omega)=0$, and

$\psi_{i j} \rightarrow \eta_{i j} \equiv \varepsilon_{1 i j}+\varepsilon_{2 i j}+\varepsilon_{3 i j}=\left(\begin{array}{rrr}0 & 1 & -1 \\ -1 & 0 & 1 \\ 1 & -1 & 0\end{array}\right)$.

We see that the undamaged state does not yield an isotropic tensor, because the components of $\boldsymbol{\eta}$ in (3.17) transform under the change of the coordinate system.

Thus the bi-vector $\psi$ defined by $(3.13 \mathrm{a}, \mathrm{b})$ is not suitable to describe the state of continuity of a damaged continuum, and we have to find another tensor composed by the bi-vectors $(3.12 \mathrm{a}, \mathrm{b}, \mathrm{c})$. As shown below, a suitable tensor of continuity may be defined by

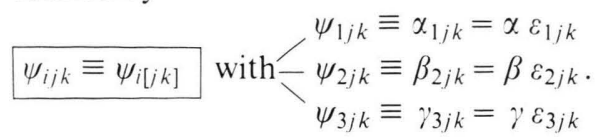

This tensor is skew-symmetric only with respect to the two bracketed indizes $[j k]$ and possesses three essential components $(\alpha, \beta, \gamma)$, as illustrated in Figure 2.

In the isotropic damage state $(\alpha=\beta=\gamma=\chi)$ the tensor (3.18) is total skew-symmetric, and the undamaged continuum $(\alpha=\beta=\gamma=1)$ is characterized by the third-order alternating tensor $\varepsilon_{i j k}$.

Supplementary to (3.18) we introduce the "damage-tensor"

$$
\begin{aligned}
&\left(_{i j k} \equiv \varepsilon_{i j k}-\psi_{i j k}\right. \\
& \text { where }-\omega_{1 j k}=(1-\alpha) \varepsilon_{1 j k}, \\
& \omega_{3 j k}=(1-\beta) \varepsilon_{2 j k}, \\
& \omega_{3 j k}=(1-\gamma) \varepsilon_{3 j k}
\end{aligned}
$$

By analogy of $(3.1 \mathrm{~b})$ or $(3.4 \mathrm{a}, \mathrm{b})$ the dual relations

$$
\begin{aligned}
& \psi_{i j k} \equiv \psi_{i[j k]}=\varepsilon_{j k r} \psi_{i r} \Leftrightarrow \psi_{i r}=\frac{1}{2} \varepsilon_{r j k} \psi_{i j k}, \\
& \omega_{i j k} \equiv \omega_{i[j k]}=\varepsilon_{j k r} \omega_{i r} \Leftrightarrow \omega_{i r}=\frac{1}{2} \varepsilon_{r j k} \omega_{i j k}
\end{aligned}
$$

are valid.

Contrary to (3.13) and (3.14) the dual tensor of continuity $\psi_{i j}$ according to (3.20) and the dual damage tensor $\psi_{i j}$ according to (3.21) have the diagonal forms

$$
\psi_{i j}=\operatorname{diag}\{\alpha, \beta, \gamma\}
$$

and

$$
\omega_{i j}=\operatorname{diag}\{(1-\alpha),(1-\beta),(1-\gamma)\},
$$

respectively. For the undamaged continuum $\left(\psi_{i j k} \rightarrow\right.$ $\varepsilon_{i j k}$ ) the dual tensor of continuity $\psi_{i j}$ is equal to Kronecker's tensor $\delta_{i j}$, as we can see from (3.20) or immediately from (3.22). The relations (3.20) and (3.22) are illustrated in Figure 2.

Especially, from Fig. 2 we can see the skew-symmetric character of the third order tensor of continuity indicated in (3.20) and its three essential components $\alpha, \beta, \gamma$. These values are fractions which represent the net cross-section elements perpendicular to the coordinate axes $x_{1}, x_{2}, x_{3}$ (Fig. 1b) and which can be measured in tests on specimens cut along three mutually perpendicular directions $x_{1}$, $x_{2}, x_{3}$.

According to $(3.4 \mathrm{a})$ a damaged surface element $\mathrm{d} \hat{S}$ can be expressed in the dual form

$$
\mathrm{d} \hat{S}_{i j}=\varepsilon_{i j k} \mathrm{~d} \hat{S}_{k} \Leftrightarrow \mathrm{d} \hat{S}_{i}=\frac{1}{2} \varepsilon_{i j k} \mathrm{~d} \hat{S}_{j k},
$$

and using the tensor of continuity (3.18) we find

$$
\mathrm{d} \hat{S}_{i j}=\psi_{k i j} \mathrm{~d} S_{k} \Leftrightarrow \mathrm{d} \hat{S}_{i}=\frac{1}{2} \psi_{i j k} \mathrm{~d} S_{j k} .
$$

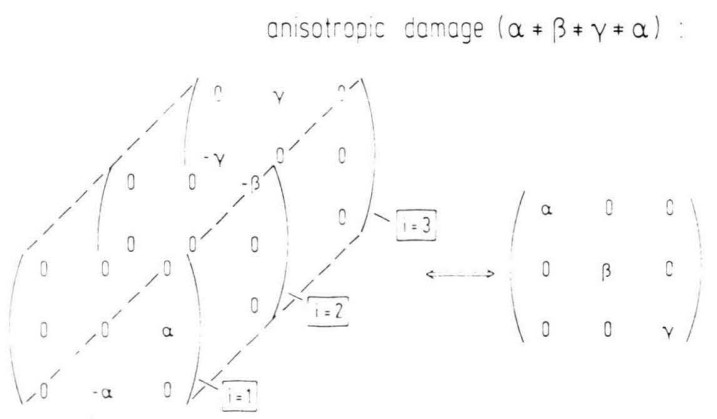

Fig. 2. Third order tensor of continuity and its dual form. 
Denote, that the bi-vector $\mathrm{d} \hat{S}_{i j}$ or $\mathrm{d} S_{j k}$ in (3.25) must have the same indizes with respect to which the tensor (3.18) is skew-symmetric. Combining (3.4a) and (3.25) we have the linear transformations

$$
\mathrm{d} \hat{S}_{i j}=\frac{1}{2} \psi_{i j p q} \mathrm{~d} S_{p q}, \quad \mathrm{~d} \hat{S}_{i}=\psi_{i r} \mathrm{~d} S_{r}, \quad(3.26 \mathrm{a}, \mathrm{b})
$$

where $\psi_{i r}$ is the tensor (3.20), (3.22) and $\psi_{i j p q}$ is a fourth-order non-symmetric tensor defined as

$$
\psi_{i j p q} \equiv \psi_{k i j} \varepsilon_{k p q},
$$

which, by using (3.20), can be expressed through

$$
\begin{aligned}
\psi_{i j p q}= & \left(\delta_{i p} \delta_{j q}-\delta_{i q} \delta_{j p}\right) \psi_{r r}-\left(\psi_{i p} \delta_{j q}-\psi_{i q} \delta_{j p}\right) \\
& -\left(\delta_{i p} \psi_{j q}-\delta_{i q} \psi_{j p}\right) .
\end{aligned}
$$

This tensor has the antisymmetric properties

$$
\psi_{i j p q}=-\psi_{j i p q}=-\psi_{i j q p}=\psi_{j i q p}
$$

and is symmetric only with respect to the index pairs, i.e.

$$
\psi_{i j p q}=\psi_{p q i j} .
$$

More briefly, the properties (3.28) and (3.29) can be indicated by

$$
\psi_{i j p q}=\psi_{([i j][p q])} .
$$

The essential components of the tensor (3.27) are given by

$\psi_{i j p q}= \begin{cases}\alpha, \beta, \gamma, & \text { if } i j \text { is an even permutation of } p q \\ -\alpha,-\beta,-\gamma, & \text { if } i j \text { is an odd permutation of } p q \\ 0, & \text { otherwise }\end{cases}$

that means

$\psi_{2323}=\psi_{3232} \equiv \alpha ; \quad \psi_{3131}=\psi_{1313} \equiv \beta ;$

$\psi_{1212}=\psi_{2121} \equiv \gamma$

$\psi_{3223}=\psi_{2332} \equiv-\alpha ; \psi_{1331}=\psi_{3113} \equiv-\beta ;$

$\psi_{2112}=\psi_{1221} \equiv-\gamma$.

In the isotropic damage state $(\alpha=\beta=\gamma=\chi)$ the tensor (3.27) is proportional to Kronecker's generalized delta $\delta_{i j p q} \equiv \varepsilon_{k i j} \varepsilon_{k p q}$, and is identical to that one in the undamaged continuum characterized by $\alpha=\beta=\gamma=1$.

\section{Stresses in a Damaged Continuum}

In the undamage continuum (Fig. 3a) Cauchy's formula

$$
p_{i}=\sigma_{j i} n_{j}
$$

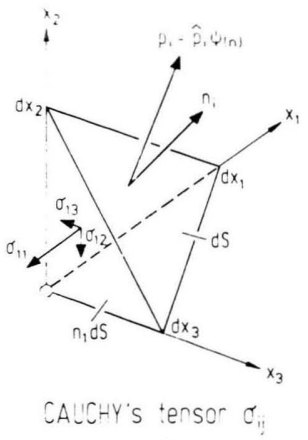

C.

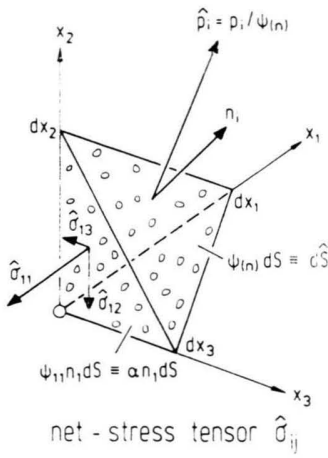

b)
Fig. 3. Stress Tensor regarding a) an undamaged, b) a damaged continuum.

is derived from equilibrium, where $p_{i}$ and $n_{i}$ are the components of the stress vector $\boldsymbol{p}$ and the unit vector normal $\boldsymbol{n}$, respectively.

In the same way we get to the corresponding relation for a damaged continuum,

$$
\hat{p}_{i} \psi_{(n)}=\psi_{j k} \hat{\sigma}_{k i} n_{j},
$$

where $\psi_{j k}$ are the components of the continuity tensor $\psi$ according to (3.20).

The surface elements $\mathrm{d} S$ and $\mathrm{d} \hat{S}$ in Fig. 3 are subjected by the same force vector:

$$
\mathrm{d} P_{i}=p_{i} \mathrm{~d} S \equiv \hat{p}_{i} \mathrm{~d} \hat{S}=\mathrm{d} \hat{P}_{i} .
$$

Thus, considering (4.1) and (4.2), we finally find the actual net-stress tensor $\hat{\boldsymbol{\sigma}}$ as a transformation from Cauchy's tensor:

$$
\sigma_{i j}=\psi_{i r} \hat{\sigma}_{r j}=\sigma_{j i} \Leftrightarrow \hat{\sigma}_{i j}=\psi_{i r}^{(-1)} \sigma_{r j} \neq \hat{\sigma}_{j i} .
$$

By suitable transvections we find $\sigma_{i j} \hat{\sigma}_{j k}^{(-1)}=\psi_{i k}$ and $\hat{\sigma}_{i j} \sigma_{j k}^{(-1)}=\psi_{i k}^{(-1)}$.

As indicated in (4.4), the actual net-stress tensor $\hat{\sigma}$ is non-symmetric, unless we have isotropic damage expressed by $\psi_{i r}=\psi \delta_{i r}$.

Because of the symmetry $\sigma_{i j}=\left(\sigma_{i j}+\sigma_{j i}\right) / 2$ of Cauchy's stress tensor $\sigma$ we find the representations

$\sigma_{i j}=\frac{1}{2}\left(\psi_{i p} \delta_{j q}+\delta_{i q} \psi_{j p}\right) \hat{\sigma}_{p q} \equiv \varphi_{i j p q} \hat{\sigma}_{p q}$,

$\hat{\sigma}_{i j}=\frac{1}{2}\left(\psi_{i p}^{(-1)} \delta_{j q}+\psi_{i q}^{(-1)} \delta_{j p}\right) \sigma_{p q} \equiv \Phi_{i j p q} \sigma_{p q}$

from (4.4). We see, the fourth-order tensors $\varphi$ and $\Phi$ defined as $(4.5 \mathrm{a}, \mathrm{b})$ are only symmetric with respect to two indices:

$$
\varphi_{i j p q}=\varphi_{j i p q}, \quad \Phi_{i j p q}=\Phi_{i j q p},
$$


that is, the actual net-stress tensor $\hat{\boldsymbol{\sigma}}$ is non-symmetric in the anisotropic damage case:

$\hat{\sigma}_{12} / \hat{\sigma}_{21}=\beta / \alpha, \quad \hat{\sigma}_{23} / \hat{\sigma}_{32}=\gamma / \beta, \quad \hat{\sigma}_{31} / \hat{\sigma}_{13}=\alpha / \gamma$.

This fact is a disadvantage, and it is awkward to use the actual net-stress tensor $\hat{\boldsymbol{\sigma}}$ in constitutive equations with a symmetric strain rate tensor $\dot{\boldsymbol{\varepsilon}}$. Therefore, we introduce a transformed net-stress tensor $\boldsymbol{t}$ defined by the operation

$$
t_{i j}=\frac{1}{2}\left(\hat{\sigma}_{i k} \psi_{k j}^{(-1)}+\psi_{k i}^{(-1)} \hat{\sigma}_{j k}\right),
$$

which is symmetric. Inserting (4.5b) into (4.8) we have

$$
t_{i j}=C_{i j p q}^{(-1)} \sigma_{p q},
$$

where

$$
C_{i j p q}^{(-1)}=\frac{1}{2}\left(\psi_{i p}^{(-1)} \psi_{j q}^{(-1)}+\psi_{i q}^{(-1)} \psi_{j p}^{(-1)}\right)
$$

is a symmetric fourth-order tensor

$$
C_{i j p q}^{(-1)}=C_{j i p q}^{(-1)}=C_{i j q p}^{(-1)}=C_{p q i j}^{(-1)} .
$$

In the undamaged $(\psi \rightarrow \delta)$ and total damaged state $(\boldsymbol{\psi} \rightarrow \boldsymbol{O})$ we have

$$
C_{i j p q}^{(-1)} \rightarrow E_{i j p q} \Rightarrow t_{i j} \rightarrow \sigma_{i j}
$$

and

$$
C_{i j p q}^{(-1)} \rightarrow \infty_{i j p q} \Rightarrow t_{i j} \rightarrow \infty_{i j} \text { (singular), }
$$

respectively, where

$$
E_{i j p q}=\frac{1}{2}\left(\delta_{i p} \delta_{j q}+\delta_{i q} \delta_{j p}\right)
$$

is the zero power tensor of rank four.

The inverse from of (4.9) is given by

$$
\sigma_{i j}=C_{i j p q} t_{p q},
$$

where

$$
C_{i j p q}=\frac{1}{2}\left(\psi_{i p} \psi_{j q}+\psi_{i q} \psi_{j p}\right)
$$

is a symmetric fourth-order tensor of continuity,

$$
C_{i j p q}=C_{j i p q}=C_{i j q p}=C_{p q i j},
$$

which is connected with the tensor (4.10) by the relation

$$
C_{i j p q} C_{p q k l}^{(-1)}=C_{i j p q}^{(-1)} C_{p q k l}=C_{i j k l}^{(0)} \equiv E_{i j k l} .
$$

Because of the symmetry properties (4.11) and (4.17) the fourth-order tensor of continuity (4.16) and its inversion (4.10) can be represented by $6 \times 6$ square matrices, which, because of (3.22), have the diagonal forms:

$C_{i j k l}=\operatorname{diag}\left\{C_{1111}, C_{2222}, C_{3333}, C_{1212}, C_{2323}, C_{3131}\right\}$

$C_{i j k l}=\operatorname{diag}\left\{\alpha^{2}, \beta^{2}, \gamma^{2}, \frac{1}{2} \alpha \beta, \frac{1}{2} \beta \gamma, \frac{1}{2} \gamma \alpha\right\}$,

and

$C_{i j k l}^{(-1)}=\operatorname{diag}\left\{C_{1111}^{(-1)}, C_{2222}^{(-1)}, C_{3333}^{(-1)}, C_{1212}^{(-1)}, C_{2323}^{(-1)}, C_{3131}^{(-1)}\right\}$

$C_{i j k l}^{(-1)}=\operatorname{diag}\left\{\frac{1}{\alpha^{2}}, \frac{1}{\beta^{2}}, \frac{1}{\gamma^{2}}, \frac{1}{2 \alpha \beta}, \frac{1}{2 \beta \gamma}, \frac{1}{2 \gamma \alpha}\right\}$,

that is, the components of the pseudo-net-stress tensor $\boldsymbol{t}$ are given in the following manner:

$t_{11}=\frac{1}{\alpha^{2}} \sigma_{11}, \quad t_{12}=\frac{1}{\alpha \beta} \sigma_{12}, \quad t_{13}=\frac{1}{\alpha \gamma} \sigma_{13}$,

$t_{21}=t_{12}, \quad t_{22}=\frac{1}{\beta^{2}} \sigma_{22}, \quad t_{23}=\frac{1}{\beta \gamma} \sigma_{23}$,

$t_{31}=t_{13}, \quad t_{32}=t_{23}, \quad t_{33}=\frac{1}{\gamma^{2}} \sigma_{33}$.

The results (4.9) and (4.15) can also be found in the following way. Using the linear transformations

$$
t_{i j}=\frac{1}{2}\left(\delta_{i r} \psi_{j s}^{(-1)}+\psi_{i s}^{(-1)} \delta_{j r}\right) \hat{\sigma}_{r s}
$$

and

$$
\hat{\sigma}_{p q}=\frac{1}{2}\left(\delta_{p r} \psi_{q s}+\delta_{p s} \psi_{q r}\right) t_{r s},
$$

which connect a fictitious symmetric tensor $\boldsymbol{t}$ with the actual non-symmetric net stress tensor $\hat{\boldsymbol{\sigma}}$, we immediately find (4.9) by inserting (4.5b) into (4.22) and (4.15) by inserting (4.23) into ( $4.5 \mathrm{a})$, respectively.

Because of the non-symmetric property of the actual net-stress tensor we find from (4.23) the decomposition

$$
\hat{\sigma}_{p q}=\hat{\sigma}_{(p q)}+\hat{\sigma}_{[p q]},
$$

where the symmetric and antisymmetric parts are given by

$$
\hat{\sigma}_{(p q)}=\left(t_{p r} \psi_{r q}+\psi_{p r} t_{r q}\right) / 2
$$

and

$$
\hat{\sigma}_{[p q]}=\left(t_{p r} \psi_{r q}-\psi_{p r} t_{r q}\right) / 2,
$$

respectively. In the special case of isotropic damage $\left(\psi_{i j}=\psi \delta_{i j}\right)$ we have $\hat{\sigma}_{(p q)}=\psi t_{p q}$ and $\hat{\sigma}_{[p q]}=O_{p q}$.

An interpretation of the introduced pseudo-netstress tensor (4.8) can be given in the following way. 
An alternative form of Cauchy's formula (4.1) is

$$
\mathrm{d} P_{i}=\sigma_{j i} \mathrm{~d} S_{j},
$$

where $\mathrm{d} P_{i}$ is the actual force vector (4.3), and according to (3.26b) we can write

$$
\mathrm{d} P_{i}=\sigma_{j i} \psi_{j r}^{(-1)} \mathrm{d} \hat{S}_{r}
$$

or inserting (4.15) we find the relation

$$
\mathrm{d} P_{i}=\psi_{i p} t_{p, r} \mathrm{~d} \hat{S}_{r},
$$

which can be multiplied by $\psi_{k i}^{(-1)}$, so that we have $\psi_{k i}^{(-1)} \mathrm{d} P_{i}=t_{k r} \mathrm{~d} \hat{S}_{r}$, or after changing the indices:

$$
\psi_{i k}^{(-1)} \mathrm{d} P_{k} \equiv \mathrm{d} \tilde{P}_{i}=t_{j i} \mathrm{~d} \hat{S}_{j} .
$$

Comparing (4.27) and (4.29) we see, that (4.29) can be interpreted as Cauchy's formula for the damaged configuration, which is subjected to the pseudoforce $\mathrm{d} \tilde{P}_{i} \equiv \psi_{i k}^{(-1)} \mathrm{d} P_{k}$ instead to the actual force $\mathrm{d} P_{i}$.

Because of the non-symmetric properties of the "net-stress-tensor" $\hat{\boldsymbol{\sigma}}$ and the operator $\boldsymbol{\varphi}$, i.e.

$$
\hat{\sigma}_{i j}=\frac{1}{2}\left(\hat{\sigma}_{i j}+\hat{\sigma}_{j i}\right)+\frac{1}{2}\left(\hat{\sigma}_{i j}-\hat{\sigma}_{j i}\right)
$$

and

$\varphi_{i j p q}=\frac{1}{2}\left(\varphi_{i j p q}+\varphi_{i j q p}\right)+\frac{1}{2}\left(\varphi_{i j p q}-\varphi_{i j q p}\right)$,

respectively, we find, from $(4.5 \mathrm{a})$, the decompositions:

$$
\begin{aligned}
\sigma_{i j}= & \frac{1}{4}\left(\varphi_{i j p q}+\varphi_{i j q p}\right)\left(\hat{\sigma}_{p q}+\hat{\sigma}_{q p}\right) \\
& +\frac{1}{4}\left(\varphi_{i j p q}-\varphi_{i j q p}\right)\left(\hat{\sigma}_{p q}-\hat{\sigma}_{q p}\right), \\
\sigma_{i j}= & \frac{1}{8}\left(\psi_{i p} \delta_{j q}+\psi_{j p} \delta_{i q}+\psi_{i q} \delta_{j p}+\psi_{j q} \delta_{i p}\right)\left(\hat{\sigma}_{p q}+\hat{\sigma}_{q p}\right) \\
& +\frac{1}{8}\left(\psi_{i p} \delta_{j q}+\psi_{j p} \delta_{i q}-\psi_{i q} \delta_{j p}-\psi_{j q} \delta_{i p}\right)\left(\hat{\sigma}_{p q}-\hat{\sigma}_{q p}\right) .
\end{aligned}
$$

Because of $(4.6 \mathrm{a})$ the right hand sides in $(4.32 \mathrm{a}, \mathrm{b})$ are symmetric with respect to the indices $i$ and $j$. Furthermore, we see the symmetry with respect to the indices $p$ and $q$. This fact can be seen immediately from (4.5a). In the special case of isotropic damage, i.e. $\psi_{i j}=\psi \delta_{i j}$ or $\hat{\sigma}_{p q}=\hat{\sigma}_{q p}$, the second term of the right hand side in $(4.32 \mathrm{~b})$ vanishes. Then, equation $(4.32 \mathrm{~b}$ ) is identical to those formulated by Rabotnov [7].

In a similar way, from (4.5b) we find the decomposition of the "net-stress-tensor" $\hat{\boldsymbol{\sigma}}$ into a symmetric and an antisymmetric part:

$$
\begin{aligned}
\hat{\sigma}_{i j}= & \frac{1}{2}\left(\Phi_{i j p q}+\Phi_{j i p q}\right) \sigma_{p q}+\frac{1}{2}\left(\Phi_{i j p q}-\Phi_{j i p q}\right) \sigma_{p q}, \\
\hat{\sigma}_{i j}= & \frac{1}{4}\left(\psi_{i p}^{(-1)} \delta_{j q}+\psi_{i q}^{(-1)} \delta_{j p}+\psi_{j p}^{(-1)} \delta_{i q}+\psi_{j q}^{(-1)} \delta_{i p}\right) \sigma_{p q} \\
& +\frac{1}{4}\left(\psi_{i p}^{(-1)} \delta_{j q}+\psi_{i q}^{(-1)} \delta_{j p}-\psi_{j p}^{(-1)} \delta_{i q}\right. \\
& \left.-\psi_{j q}^{(-1)} \delta_{i p}\right) \sigma_{p q} .
\end{aligned}
$$

The results given above may be expressed by the damage tensor $\omega$. For instance, from $(3.27 \mathrm{a}, \mathrm{b})$ in connection with (3.19) and because of $\psi_{i j} \equiv \delta_{i j}-\omega_{i j}$ we have

$$
\begin{aligned}
\psi_{i j p q}= & \delta_{i j p q}-\omega_{k i j} \varepsilon_{k p q} \equiv\left(\varepsilon_{k i j}-\omega_{k i j}\right) \varepsilon_{k p q}, \\
\psi_{i j p q}= & \left(\delta_{i p} \delta_{j q}-\delta_{i q} \delta_{j p}\right)\left(1-\omega_{r r}\right)+\left(\omega_{i p} \delta_{j q}-\omega_{i q} \delta_{j p}\right) \\
& +\left(\delta_{i p} \omega_{j q}-\delta_{i q} \omega_{j p}\right) .
\end{aligned}
$$

Furthermore, instead of (4.5a) and (4.32 b) we find

$\sigma_{i j}=\frac{1}{2}\left[\delta_{i p} \delta_{j q}+\delta_{i q} \delta_{j p}-\left(\omega_{i p} \delta_{j q}+\delta_{i q} \omega_{j p}\right)\right] \hat{\sigma}_{p q}$

and

$$
\begin{aligned}
\sigma_{i j}= & \frac{1}{2}\left(\hat{\sigma}_{i j}+\hat{\sigma}_{j i}\right) \\
& -\frac{1}{8}\left(\omega_{i p} \delta_{j q}+\delta_{i q} \omega_{j p}+\omega_{i q} \delta_{j p}+\delta_{i p} \omega_{j q}\right)\left(\hat{\sigma}_{p q}+\hat{\sigma}_{q p}\right) \\
& -\frac{1}{8}\left(\omega_{i p} \delta_{j q}+\delta_{i q} \omega_{j p}-\omega_{i q} \delta_{j p}-\delta_{i p} \omega_{j q}\right)\left(\hat{\sigma}_{p q}-\hat{\sigma}_{q p}\right) .
\end{aligned}
$$

By using the inverse

$$
\psi_{i r}^{(-1)} \equiv \frac{1}{2 \operatorname{det}(\psi)} \varepsilon_{r q p} \varepsilon_{i k l} \psi_{p k} \psi_{q l}
$$

and because of the symmetry $\sigma_{i j}=\left(\sigma_{i j}+\sigma_{j i}\right) / 2$, we find the following relations for the "net-stresstensor":

$$
\begin{gathered}
\hat{\sigma}_{i j}=\frac{1}{2 \operatorname{det}(\boldsymbol{\delta}-\omega)}\left[\left(\delta_{i s} \delta_{j t}+\delta_{i t} \delta_{j s}\right)\left(1-\omega_{r r}\right)\right. \\
+\left(\omega_{i s} \delta_{j t}+\omega_{i t} \delta_{j s}\right) \\
+\frac{1}{2} \varepsilon_{i k l}\left(\varepsilon_{s p q} \delta_{j t}\right. \\
\left.\left.+\varepsilon_{t p q} \delta_{j s}\right) \omega_{p k} \omega_{q l}\right] \sigma_{s t},
\end{gathered}
$$

where

$J_{1}(\boldsymbol{\omega}) \equiv \delta_{i j} \omega_{j i}, \quad J_{2}(\boldsymbol{\omega}) \equiv \frac{1}{2}\left(\omega_{i j} \omega_{j i}-\omega_{i i} \omega_{j j}\right)$

are invariants of the damage tensor $\omega$.

Finally, we consider Cauchy's stress equations of equilibrium,

$$
\sigma_{j i, j}=0,
$$

in the absence of body forces. Then, by using the transformation (4.4), we have the equilibrium equations in the net stresses:

$$
\hat{\sigma}_{r i} \psi_{j r, j}+\psi_{j r} \hat{\sigma}_{r i, j}=0 .
$$


The symmetry of Cauchy's stress tensor $\left(\sigma_{i j}=\sigma_{j i}\right)$ resulting from moment equilibrium yields the condition

$\psi_{i p} \hat{\sigma}_{p j}=\psi_{j q} \hat{\sigma}_{q i} \quad$ or $\quad \hat{\sigma}_{i j}=\psi_{i q}^{(-1)} \psi_{j p} \hat{\sigma}_{p q}$

which states, that the "net-stress tensor" is non symmetric. From $(4.41 \mathrm{~b})$ we find the decomposition

[1] M. Chrzanowski, The Description of Metallic Creep in the Light of Damage Hypothesis and Strain Hardening. Diss. hab., Politechnika Krakowska, Krakow 1973.

[2] R. P. Goel, J. Appl. Mech., Transactions of the ASME, September 1975 , pp. 625-629.

[3] D. R. Hayhurst and F. A. Leckie, J. Mech. Phys. Solids 21, 431 (1973).

[4] D. R. Hayhurst, W. A. Trampczynski, and F. A. Leckie, Acta Metallurgica Vol. 28, 1171 (1980). into a symmetric part and an antisymmetric one:

$$
\begin{aligned}
\hat{\sigma}_{i j}= & \frac{1}{4}\left(\psi_{i q}^{(-1)} \psi_{j p}+\psi_{i p}^{(-1)} \psi_{j q}\right)\left(\hat{\sigma}_{p q}+\hat{\sigma}_{q p}\right) \\
& +\frac{1}{4}\left(\psi_{i q}^{(-1)} \psi_{j p}-\psi_{i p}^{(-1)} \psi_{p q}\right)\left(\hat{\sigma}_{p q}-\hat{\sigma}_{q p}\right) .
\end{aligned}
$$

For the isotropic damage case $\left(\psi_{i j}=\psi \delta_{i j}\right)(4.42)$ is equal to the decomposition $\hat{\sigma}_{i j}=\left(\hat{\sigma}_{j i}+\hat{\sigma}_{i j}\right) / 2+$ $\left(\hat{\sigma}_{j i}-\hat{\sigma}_{i j}\right) / 2$, i.e. the "net-stress tensor" is symmetric $\left(\hat{\sigma}_{i j}=\hat{\sigma}_{j i}\right)$ in this special case only.

[5] S. Murakami and N. Ohno, Inelastic Behaviour of Pressure Vessel and Piping Components (Edited by $\mathrm{T}$. Y. Chang and E. Krempl), PVP-PB-028, ASME, New York 1978, pp. 55-69.

[6] S. Murakami and N. Ohno, Presentation at the 3rd IUTAM Symposium on Creep in Structures, Leicester, September 8-12, 1980, published in the proceedings.

[7] Y. N. Rabotnov, Proceedings, Applied Mechanics Conference, Stanford University, edited by M. Hetenyi and H. Vincenti, 1968, pp. 342. 